\title{
PROVAS DE FUNÇÃO PULMONAR NO PRÉ E PÓS-OPERATÓRIO DE REDUÇÃO GASTRICA POR CELIOTOMIA OU POR VIDEOLAPAROSCOPIA
}

\section{PULMONARY FUNCTION TESTS IN PRE AND POSTOPERATIVE OF GASTRIC BYPASS BY LAPAROTOMY OR VIDEOLAPAROSCOPY}

\author{
Geraldo Magela Cardoso Filho'; Augusto Diogo Filho, TCBC-MG; \\ Gabriel de Camargo Cunha Ribeiro ${ }^{3}$
}

\begin{abstract}
RESUMO: Objetivo: avaliar a função pumonar no pré e pós-operatório de indivíduos submetidos a redução gastrica por celiotomia(GC) ou videolaparoscopia(GV). Método: No período de Outubro de 2004 a outubro de 2005, foram submetidos à gastroplastia por Y de Roux 17 pacientes abordados por celiotomia e 20 pacientes abordados por videolaparoscopia . Nesses pacientes foram realizados espirometria , oximetria de pulso e monitorização da frequência respiratória(FR) antes e após a cirurgia até a $30^{a}$ hora e avaliação da dor pela escala visual analógia modificada no momento da espirometria. Resultados: No préoperatório, todos os pacientes apresentaram provas de função pulmonar normais. Observou-se que do pré para o pós-operatório houve redução média significativa de 38,5\% CFV, 39,1\% VEF e $37 \%$ do PEF no Grupo GC e redução também significativa de 38,3\% de CVF, 35,5\% de VEF 1 e 41,5\% de PEF no Grupo GV. A FR apresentou aumento médio com significância estatistica de 16,9\%e

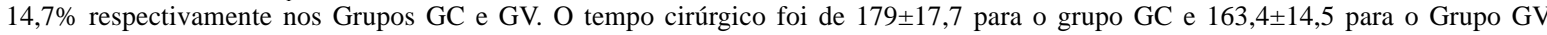
e os scores de dor 3,5 $\pm 1,2$ e 3,4 $\pm 1,5$ para GC e GV respectivamente. A incidência de complicações pós-operatórias foi de $2 \% \%$. Conclusão: concluímos que indivíduos do nosso estudo com obesidade grau II ou III apresentaram espirometrias normais e que ao se submeterem a gastroplastia pela técnica de Capella por celiotomia ou videolaparoscopia, apresentaram um padrão espirométrico de restrição pulmonar no pós-operatório imediato (Rev. Col. Bras. Cir. 2008; 35(6): 382-386).
\end{abstract}

Descritores: Testes de função respiratória; Cuidados pré-operatórios; Obesidade; Obesidade Mórbida; Cirurgia bariátrica.

\section{INTRODUÇÃO}

A obesidade é o acúmulo de tecido gorduroso, regionalizado ou em todo o corpo, de origem genética ou endócrino-metabólica ou por distúrbios nutricionais ${ }^{1}$. No Brasil é cada vez mais evidente o aumento de casos de obesidade e sobrepeso nos diferentes seguimentos da população brasileira ${ }^{2}$.

São considerados obesos indivíduos com IMC acima de $30 \mathrm{~kg}$ e classificado em Grau I, IMC de 30 a $34,9 \mathrm{Kg} / \mathrm{m}^{2}$; Grau II, IMC entre 35 a $39,9 \mathrm{Kg} / \mathrm{m}^{2}$ e obesidade Grau III ou mórbida para IMC acima de $40 \mathrm{~kg} / \mathrm{m}^{2}$, segundo a Organização Mundial de Saúde (OMS) ${ }^{3-5}$. Dentre os procedimentos cirúrgicos propostos para o tratamento da obesidade, o procedimento de Capella (redução gástrica a $30 \mathrm{ml}$ a $50 \mathrm{ml}$ com derivação em Y de Roux), apresenta o melhor índice de sucesso na redução de peso, tanto a curto como em longo prazo, e é endossado pelos institutos nacionais de saúde 5 .

Procedimentos cirúrgicos no abdome podem agravar a função pulmonar por proporcionarem aumento da pressão intra-abdominal, atelectasias e "shunt" pulmonar". Indivíduos com obesidade podem apresentar o consumo de oxi- gênio e a produção de dióxido de carbono aumentados, apnéia do sono e síndrome da hipoventilação, responsáveis pela redução dos volumes pulmonares ${ }^{7}$. Diante desses fatores, o tratamento cirúrgico em pacientes com obesidade de grandes proporções se justifica pela evidência de apresentarem menor expectativa de vida e comorbidades associadas, reforçado pelo baixo índice de sucesso nos métodos não - cirúrgicos ${ }^{8}$. Apesar da eficácia e relativa segurança da cirurgia por via convencional observa-se índices significativos de complicações. Por outro lado a cirurgia videolaparoscópica ou cirurgia minimamente invasiva revolucionou o tratamento de uma série de afecções no abdome pela menor agressão cirúrgica ${ }^{9}$.

O objetivo desse estudo foi de avaliar a função pulmonar em indivíduos obesos Grau II e/ou III no pré e pósoperatório de cirurgia de redução gástrica com reconstrução à Capella, em cada grupo: celiotomia e videolaparoscopia, separadamente.

\section{MÉTODO}

Trata-se de uma série de casos consecutivos em que se avaliou as provas de função pulmonar no pré-operatório e

1. Mestre em Ciências da Saúde pela Faculdade de Medicina da Universidade Federal de Uberlândia - UFU.

2. Titular do Colégio Brasileiro de Cirurgia Digestiva, Professor Associado do Departamento de Cirurgia da Faculdade de Medicina da Universidade Federal de Uberlândia - UFU; Professor do Programa de Pós-Graduação em Ciências da Saúde da Faculdade de Medicina da Universidade Federal de Uberlândia - UFU.

3. Acadêmico do Curso de Medicina da Universidade Federal de Uberlândia - UFU.

Recebido em 20/05/2008

Aceito para publicação em 03/09/2008

Conflito de interesses: nenhum

Fonte de financiamento: nenhuma

Trabalho realizado no Departamento de Cirurgia da Faculdade de Medicina da Universidade Federal de Uberlândia - UFU - MG 
pós-operatório imediato de pacientes submetidos à cirurgia de redução gástrica para o tratamento da obesidade graus II e III submetidos a duas formas de abordagens cirúrgicas: por via convencional (celiotomia) e por videolaparoscopia.

O estudo foi aprovado pelo CEP - UFU (Comitê de Ética em Pesquisa em Humanos da Universidade Federal de Uberlândia) e realizada no período de outubro de 2004 a outubro de 2005 .

Os pacientes foram distribuídos em grupos abordados cirurgicamente por celiotomia (18 pacientes) e por videolaparoscopia (21pacientes). Destes, 26 do sexo feminino e 13 do sexo masculino, sendo 12 em hospital público universitário e 27 em hospital privado. No hospital público a abordagem cirúrgica era apenas por celiotomia e no privado a escolha pela abordagem era do paciente. $\mathrm{O}$ pesquisador desconhecia a seqüência de aparecimento dos indivíduos durante o período.

Após a assinatura do termo de consentimento informado e esclarecido, foi preenchida uma ficha de avaliação com dados pessoais e antropométricos e realizado exames de espirometria no pré-operatório, com espirômetro portátil da marca Vitalograph, modelo 2120 e oximetria de pulso com oxímetro portátil da marca Oxiplus.

Os critérios de inclusão dos pacientes foram: idade superior a 18 anos, sem DPOC, que seriam submetidos à técnica de Capella e com obesidade Grau II ou III. Já os critérios de exclusão foram: pacientes que precisassem de ventilação mecânica no pós-operatório imediato; reintervenção cirúrgica, ou que evoluíssem com complicação pós-operatória (CPP) como pneumonia, traqueobronquite, insuficiência respiratória aguda, broncoespasmo e tromboembolismo pulmonar.

Dos 39 pacientes submetidos à avaliação pré-operatória, houve desistência de um no grupo dos operados por celiotomia e uma exclusão no grupo por videolaparoscopia por apresentar infecção respiratória no pós-operatório imediato.

Durante o exame espirométrico, o paciente era posicionado sentado e era estimulado vigorosamente para que o esforço fosse "explosivo" no início da manobra. Durante a expiração o paciente era observado e estimulado positivamente para que o esforço fosse mantido pelo tempo necessário, não excedendo oito tentativas.

Os critérios de aceitabilidade e reprodutibilidade foram os recomendados pela American Thoracic Society $(1995)^{10}$, Sociedade Brasileira de Pneumologia Tisiologia (1996) ${ }^{11}$ e Diretrizes Para Prova de Função Pulmonar (2002) ${ }^{12}$. Os Valores de referência empregados foram os de Knudson et al (1976 e $1983)^{13,14}$. Foram obtidas três curvas expiratórias tecnicamente aceitáveis no pré e pós-operatórios até a trigésima hora.

No pós-operatório até a trigésima hora, o paciente repetia a espirometria, a oximetria de pulso e ainda realizava avaliação da dor pós-operatória no momento da espirometria utilizando a escala visual analógica (EVA), modificada.

Para comparar a diferença dos valores encontrados no pré e pós-operatórios dos pacientes operados por celiotomia e por videolaparoscopia foram aplicados os testes t de Student aos valores de saturação de oxigênio, capacidade vital força$\mathrm{da}(\mathrm{CVF})$, volume expirado no primeiro segundo $\left(\mathrm{VEF}_{1}\right)$ e pico de fluxo expiratório (PEF) e de Wilcoxon aos valores de freqüência respiratória separadamente, em cada grupo. Os grupos foram analisados separadamente por não terem sido "randomizados" quanto a seqüência de abordagem cirúrgica.

Com o intuito de verificar a existência ou não de correlações estatisticamente significantes, entre os valores das variáveis idade, IMC e tempo de cirurgia com os valores de CVF, VEF ${ }_{1}, \mathrm{PEF}$, foram aplicados os Coeficientes de Correlação de Person e o Coeficiente de Correlação por Postos de Spearman aos dados relativos em cada grupo.

Os valores foram considerados estatisticamente significantes quando a probabilidade de rejeição da hipótese de nulidade foi menor do que $0,05(5 \%)$.

\section{RESULTADOS}

Com relação aos dados antropométricos, a média de idade dos 17 pacientes do grupo celiotomia foi de $32 \pm 9,8$ anos e IMC de $47 \pm 7,2 \mathrm{~kg} / \mathrm{m}^{2}$ onde os homens corresponderam a $47 \%$ dos pacientes $(\mathrm{n}=8)$ e as mulheres representaram $53 \%$ da amostra (n=9). Já no grupo videolaparoscopia a média de idade foi de $35 \pm 9,6$ anos e IMC de 44 $\pm 7,2 \mathrm{Kg} / \mathrm{m}^{2}$ sendo $35 \%$ (n=7) homens e $65 \%(n=13)$ mulheres.

As médias e o desvio padrão da freqüência respiratória (FR), saturação de oxigênio $\left(\mathrm{SatO}_{2}\right)$, capacidade vital forçada $(\mathrm{CVF})$, volume expiratório forçado no $1^{\circ}$ segundo $\left(\mathrm{VEF}_{1}\right)$ e pico de fluxo expiratório (PEF) no pré-operatório e pós-operatório e tempo de cirurgia e score de dor pela escala visual analógica pós-operatório no grupo celiotomia e no grupo videolaparoscopia estão representados respectivamente nas tabelas 1 e 2 .

A incidência de complicações pulmonares pós-operatórias neste estudo foi baixa, $(2,7 \%)$. Um paciente apresentou infecção respiratória, sem óbitos no período estudado.

\section{DISCUSSÃO}

No pré-operatório, encontramos valores espirométricos normais em relação aos previstos por Knudson para todos os pacientes. Esses achados podem ser justificados pelo período adaptativo frente ao ganho de peso e por apresentarem grande reserva energética ${ }^{15,16}$

As cirurgias no andar superior do abdome e a concentração de gordura em região tóraco - abdominal adicionado a outros fatores podem influenciar na função pulmonar dos pacientes ${ }^{14}$. Em nosso estudo, não encontramos correlação entre IMC e valores espirométricos de CVF, $\mathrm{VEF}_{1}$ e PEF no pré-operatório de ambos os grupos (celiotomia e videolaparoscopia) pelo Coeficiente de Correlação de Person. É possível que esta falta de correlação seja em decorrência do predomínio na distribuição periférica de gordura na mulher (obesidade ginecoide), uma vez que em nossa amostra houve predomínio do sexo feminino $(66,6 \%)$. Sabe-se que a distribuição de gordura tóraco - abdominal, como na obesidade androide, é um fator de risco para complicações pulmonares ${ }^{15}$. A obesidade androide interfere na descida do diafragma e na complacência pulmonar o que resulta em menores volumes pulmonares ${ }^{17,18}$. Adicionando a essas justificativas, Zerah et 
Tabela 1 - Média e desvio padrão $F R, \mathrm{SatO}_{2} C V F, V E F_{1}$, PEF no pré e pós-operatórios, tempo de cirurgia e score de dor pela escala visual analógica (EVA) no pós-operatório e a diferença em percentagem do pré para o pós-operatório de 17 pacientes submetidos à redução gástrica por abordagem cirúrgica por CELIOTOMIA.

\begin{tabular}{lcccc}
\hline Variáveis & Pré-operatório & Pós-operatório & $\begin{array}{c}\text { Ciferença em \% do Pré } \\
\text { para o Pós-operatório }\end{array}$ & Valor de $p$ \\
\hline FR $($ irpm $)$ & $14,5 \pm 1,7$ & $17,4 \pm 3,7^{*}$ & 16,9 & 0,00 \\
Sat. $\mathrm{O}_{2}(\%)$ & $95,4 \pm 1,5$ & $95,6 \pm 1,0$ & 0,5 & 0,41 \\
$\mathrm{CVF}(\mathrm{L})$ & $3,5 \pm 1,0$ & $2,3 \pm 0,7^{*}$ & 38,5 & 0,00 \\
$\mathrm{VEF}_{1}(\mathrm{~L})$ & $3,0 \pm 0,7$ & $2,0 \pm 0,6^{*}$ & 39,1 & 0,00 \\
$\mathrm{PEF}(\mathrm{L})$ & $7,8 \pm 1,8$ & $5,4 \pm 2,2^{*}$ & 37 & 0,00 \\
T. Cirurgia (Minutos) & $\ldots$ & $17,9 \pm 17,7$ & $\ldots$ & $\ldots$ \\
Escala de Dor-EVA & $\ldots$ & $3,5 \pm 1,2$ & $\ldots$ & $\ldots$ \\
\hline
\end{tabular}

FR: freqüência respiratória; Sat. $O_{2:}$ saturação de oxigênio, CVF: capacidade vital forçada; VEF1: volume expiratório forçado no $1^{o}$ segundo; PEF: pico de fluxo expiratório, ... : não há dados; irpm: incursões respiratórias por minuto $(* p<0,05)$.

al. ${ }^{19}$, apontaram diminuições da Capacidade Vital, da Capacidade Pulmonar Total, da Capacidade Residual Funcional e do Volume de Reserva Expiratório nos pacientes masculinos.

Quanto as médias das variáveis espirométricas, tanto na abordagem cirúrgica por celiotomia como por videolaparoscopia, houve redução com significância estatística em todas as variáveis: $\mathrm{CVF}_{1} \mathrm{VEF}_{1}$ e PEF (Tabelas 1 e 2). Do pré-operatório para o pós-operatório, resultados semelhantes também foram encontrados na literatura ${ }^{16}$. Essa redução pode ser explicada pela interferência do procedimento cirúrgico, uma vez que podem afetar os músculos respiratórios por uma série de mecanismos como as alterações do controle neural, da integridade muscular, dos mecanismos reflexos, alterações no comprimento-tensão, na mecânica tóraco-abdominal, o próprio anestésico, a dor pós-operatória e o procedimento cirúrgico $^{17}$.

Em nosso estudo na abordagem por celiotomia, encontrou-se redução de 38,5\% para CVF, 39,1\% para $\mathrm{VEF}_{1} \mathrm{e}$ $37 \%$ para PEF, resultados semelhantes foram encontrados num grupo de pacientes submetidos à cirurgia abdominal alta por celiotomia, sob anestesia geral, nos quais houve reduções da $\mathrm{CVF}$ em $45 \%$, da $\mathrm{VEF}_{1}$ em $48 \%$ e da capacidade residual funcional em $76 \%$ em relação aos valores pré-operatórios ${ }^{18}$. Essas reduções ocorrem em função de alterações respiratórias importantes, decorrentes: da dor, efeitos residuais dos anestésicos e miorrelaxantes, fadiga do diafragma, mudanças na conformação do gradil costo-diafragmático. Essas alterações culminam com restrição da complacência pulmonar, diminuição do volume corrente e da capacidade residual funcional, diminuição da pressão intracavitária, e da capacidade do diafragma de gerar pressões e de conter as vísceras ${ }^{19}$.

$\mathrm{Na}$ abordagem por videolaparoscopia, encontrouse na literatura, redução de até $47 \%$ dos valores previstos de CVF do pré-operatório para o primeiro dia de pós-operatório ${ }^{15}$. No nosso estudo redução foi de $38,3 \%$ para CVF, $35,5 \%$ para $\mathrm{VEF}_{1}$ e $41,5 \%$ para PEF. Por essa via de abordagem, a função respiratória pode estar comprometida durante $o$ ato operatório pela presença do pneumoperitônio com aumento da pressão intra-abdominal e elevação do diafrag-

Tabela 2 - Média e desvio padrão FR, $\mathrm{SatO}_{2}, C V F, V E F_{1}$, PEF no pré e pós-operatórios, tempo de cirurgia e score da EVA no pós-operatório e a diferença em percentagem do pré para o pós-operatório de 20 pacientes submetidos à redução gástrica por abordagem cirúrgica por videolaparoscopia.

\begin{tabular}{|c|c|c|c|c|c|}
\hline \multicolumn{6}{|c|}{ Videolaparoscopia } \\
\hline \multirow{2}{*}{$\begin{array}{l}\text { Variáveis } \\
\text { FR (irpm) }\end{array}$} & Pré-operatório & \multicolumn{2}{|c|}{ Pós-operatório } & \multirow{2}{*}{$\begin{array}{c}\begin{array}{c}\text { Diferença em \% do Pré } \\
\text { para o Pós-operatório }\end{array} \\
14,7\end{array}$} & \multirow{2}{*}{$\begin{array}{c}\text { Valor de } p \\
0,00\end{array}$} \\
\hline & $15,4 \pm 2,9$ & $17,9 \pm$ & $3,7^{*}$ & & \\
\hline Sat. $\mathrm{O}_{2}(\%)$ & $95,6 \pm \quad 1,2$ & $95,3 \pm$ & 1,3 & 0,3 & 0,47 \\
\hline $\mathrm{CVF}(\mathrm{L})$ & $3,8 \pm \quad 0,8$ & $2,3 \pm$ & $0,6^{*}$ & 38,3 & 0,00 \\
\hline $\mathrm{VEF}_{1}(\mathrm{~L})$ & $3,2 \pm 0,6$ & $2,1 \pm$ & $0,1^{*}$ & 35,5 & 0,00 \\
\hline PEF (L) & $8,3 \pm 2,1$ & $4,8 \pm$ & $1,8^{*}$ & 41,5 & 0,00 \\
\hline T. Cirurgia (Minutos) & $\ldots$ & $163,4 \pm$ & 14,5 & $\ldots$ & $\ldots$ \\
\hline Escala de Dor-EVA & ... & $3,4 \pm$ & 1,5 & $\ldots$ & $\ldots$ \\
\hline
\end{tabular}

FR: freqüência respiratória; Sat. $O_{2:}$ saturação de oxigênio, CVF: capacidade vital forçada; VEF 1: volume expiratório forçado no ${ }^{\circ}$ segundo; PEF: pico de fluxo expiratório, ... : não há dados; irpm: incursões respiratórias por minuto (*p $<0,05)$. 
ma, podendo causar redução da complacência pulmonar e limitar a expansão além da irritação do diafragmática pelo $\mathrm{CO}_{2}{ }^{2021}$.

Em estudo de 480 pacientes submetidos à cirurgia abdominal alta, os autores identificaram como fatores de risco à ocorrência de complicações pulmonares pós- operatórias e a partir daí, formularam um índice prognóstico constando de oito categorias de risco considerando a existência de pneumopatia crônica, tempo cirúrgico maior que 210 minutos e a presença de hipertensão arterial, diabetes mellitus, cardiopatia e idade avançada ${ }^{22}$.

Embora sejam poucos os estudos que abordam as complicações respiratórias pós-operatórias em obesidade mórbida em pacientes submetidos à gastroplastia, sabe-se que nas cirurgias do abdome superior, as complicações respiratórias pós-operatórias estão fortemente relacionadas à anestesia geral por necessitar de intubação endotraqueal, por provocar relaxamento muscular, que pode ocasionar a aspiração brônquica e por utilizar assistência ventilatória e redução do reflexo de tosse $\mathrm{e}^{2324}$. No presente estudo foi observado apenas um caso de complicação respiratória pósoperatória no grupo abordado por videolaparoscopia por apresentar quadro de hipersecreção pulmonar, não havendo significância estatística. A diminuição do transporte de secreção pelo uso de anestésicos, o aumento de muco pela irritação traqueal em função da intubação, diminuição da força muscular tornando a respiração mais superficial com ausência de suspiros, explica essa complicação pulmonar pósoperatória (CPP) ${ }^{25}$.

Quando analisado o tempo de cirurgia em nosso trabalho, o tempo médio para a abordagem por celiotomia foi de 179 minutos e por videolaparoscopia de 163 minutos, o que pode ter contribuído para a baixa incidência de complicações pulmonares, já que outros autores verificaram CPP com tempo cirúrgico maior que 210 minutos ${ }^{25}$.

Em relação à idade dos pacientes, em alguns estudos, a idade superior a 65 anos esteve associada ao maior numero de complicações respiratórias ${ }^{25}$. Essa variável em nosso trabalho não apresentou significância, visto que as médias de idade foram de 32,5 anos e 35,6 anos para os grupos celiotomia e videolaparoscopia respectivamente.

A analgesia pós-operatoria adequada pode ter contribuído pela presença de dor de leve a moderada intensidade na avaliação de score da Eva. Joris (1997) em estudo onde comparou a intensidade da dor do pós-operatório de gastroplastia por celiotomia com a dor do pós-operatório de gastroplastia por videolaparoscopia, concluiu que no período imediato até a $18^{\mathrm{a}}$ hora do primeiro dia pós-operatório, a intensidade da dor manteve-se de leve a moderada nas duas abordagens ${ }^{26}$. É importante ressaltar que ao correlacionar a média de dor com as médias de todas as variáveis do nosso estudo no pós-operatório, observou-se que não houve correlação estatística.

A medida da oximetria de pulso do pré-operatório para o pós-operatório foi semelhante em ambos os grupos. Resultados semelhantes foram encontrados por Chiavegato et al. ${ }^{27}$. A gênese das alterações na saturação de oxigênio no período pós-operatório é multifatorial e engloba a sinergia entre a doença do paciente, os efeitos da anestesia, a área cirúrgica, as alterações causadas pelo procedimento cirúrgico, idade dos pacientes e o tipo de analgesia empregada no pós-operatório. Estes fatores podem contribuir em baixos volumes inspiratórios ${ }^{27}$, o que também explica o aumento da frequência respiratória no pós-operatório nos dois grupos como forma de compensação frente ao padrão respiratório superficial ${ }^{27}$.

Em nossos casos podemos concluir que indivíduos com obesidade Grau II ou III apresentam espirometrias normais e que ao se submeterem à cirurgia bariátrica pela técnica de Capella tanto por celiotomia como por videolaparoscopia, apresentam um padrão espirométrico de restrição pulmonar no pós-operatório imediato. Conclui-se também que a incidência de complicações pulmonares pós-operatória nesta população estudada foi baixa.

\begin{abstract}
Background: Evaluate pulmonary function pre and postoperative period of patients submitted to Roux-en-Y gastric bypass by laparotomy and video laparoscopy. Method: Between october 2004 until october 2005, 38 patients were submitted to Roux-en-Y gastric bypass, 17 of them ( 9 woman and 8 man) by laparotomy (age and BMI mean of 32 $\pm 9,8$ years and $47 \pm 7,2 \mathrm{Kg} / \mathrm{m}^{2}$, respectively) and 20 (13 woman and $7 \mathrm{man}$ ) by video laparoscopy (age and BMI mean of 35 $\pm 9,6 y e a r s$ and $44 \pm 5,9 \mathrm{Kg} / \mathrm{m}^{2}$, respectively). All of them were submitted to espirometry, blood gas transcutaneous (oximetry) and respiratory frequency monitoring before and after surgery (until the $30^{\text {th }}$ hour) and were also evaluated by a modified pain visual analogic scale (VAS) at the espirometry. Results: In pre-operative, all patients presented normal pulmonary function tests. We observed a significant mean reduction of 38,5\% in FVC, 39,11\% in FEV1 and 37\% in PEF in laparotomy group (LG). In the video laparoscopy group $(V G)$ there was a mean reduction of $38.37 \%$ in FVC, $35.53 \%$ in FEV1 and $41.57 \%$ in PEF. The respiratory frequency had a mean increase of 16.98 and $14.79 \%$ in $L G$ and VG respectively, both with statistic significance. The mean operating time was 179 17,7 minutes in $L G$ and 163,4 $\pm 14,5$ minutes in VG. Pain scores were 3.5 in $L G$ and 3.40 in VG. The incidence of post-operatory complications was about $2 \%$.Conclusion: We concluded that the patients with degree 2 or morbid obesity presented normal espirometry values in pre-operative and, after being submitted to gastric bypass technique (laparotomy or videolaparoscopy), they developed an espirometric standard compatible with pulmonary restriction in early post-operative.
\end{abstract}

Key words: Respiratory Function Tests; Preoperative Care; Obesity, Morbid; Obesity; Bariatric surgery. 


\section{REFERÊNCIAS}

1. Fisberg M. Atualização em obesidade na infância e adolescência. São Paulo: Ateneu; 2005.

2 Guedes DP, Guedes JE. Controle do peso corporal; composição corporal, atividade física e nutrição. Rio de Janeiro: Shape; 2003.

3. Auler JR, Giannini CG, Saraiotto DF. Desafios no manuseio peri-operatório de pacientes obesos mórbidos: como previnir complicações . Rev Bras Anestesiol. 2003; 53(2):227-36.

4. Bender R, Jöckel KH, Richter B, Sprual M, Berger M. Body weight, blood pressure, and mortality in cohort of obese patients. Am J Epidemiol. 2002; 156(3):239-45.

5. Consenso Latino-Americano de Obesidade. Arq Bras Endocrinol Metab. 1999; 43(1):21-67.

6. Garrido JAB. Situações especiais: tratamento da obesidade mórbida. In: Halpern A, Godoy M, Suplicy HL, Mancini MC, Zanella MT. Obesidade. São Paulo: Lemos Editorial; 1998. p. 331-41.

7. Coussa M, Proietti S, Schnyder P, Frascarolo P, Suter M, Spahn DR, Magnusson L. Prevention of atelectasis formation during the induction of general anesthesia in morbidly obese patients. Anesth Analg. 2004; 98(5):1491-5.

8. Vaughan RW, Cork RC, Hollander D. The effect of massive weight loss on arterial oxygenation and pulmonary function tests. Anesthesiology. 1981; 54(4):325-8.

9. Balsiger BM, Murr MM, Poggio JL. Bariatric surgery. Surgery for weight control in patients with morbid obesity. Med Clin North Am. 2000; 84(2):477-89.

10. Morino M, Toppino M, Bonnet G, del Genio G. Laparoscopic adjustable silicone gastric banding versus vertical banded gastroplasty in morbidly obese patients: a prospective randomized controlled clinical trial. Ann Surg. 2003; 238(6):835-41; discussion 841-2.

11. Standardization of Spirometry-1987 update. Statement of the American Thoracic Society. Am Rev Respir Dis. 1987;136(5):1285-98.

12. Sociedade Brasileira de Pneumologia e Tisiologia. Diretrizes para Testes de Função Pulmonar. J Pneumol. 2002; 28 Suppl 3: S1S238.

13. Pereira CAC, Neder JA. Diretrizes para testes de função pulmonar. J Pneumol. 2002; 28 Suppl 3: S1-S41.

14. Knudson RJ, Slatin RC, Lebowitz MD, Burrows B. The maximal expiratory flow-volume curves. Normal standards, variability, and effects of age. Am Rev Respir Dis. 1976;113(5):587-600.

15. Knudson RJ, Lebowitz MD, Holberg CJ, Burrows B. Changes in the maximal expiratory flow-volume curve with growth and aging. Am Rev Respir Dis. 1983; 127(6):725-34.

16. Paisani DM, Chiavegato LD, Faresin SM. Volumes, capacidades pulmonares e força muscular respiratória no pós-operatório de gastroplastia. J Bras Pneumol. 2005; (2):125-32.
17. Siafakas NM, Mitrouska I, Bourus D,Georgopoulos D. Surgery and the respiratory muscles. Thorax. 1999;54(5):458-65.

18. Holley HS, Millic-Emili J, Bechlake MR, Bates DV. Regional distribuition of pulmonary ventilation and perfusion in obesity. J. Clin Invest. 1967;46(4):475-81.

19. Koenig SM. Pulmonary complications of obesity. Am J Med Sci. 2001; 321(4):249 - 79.

20. Zerah F, Harf A, Perlemuter L, Lorino H, Lorino AM, Atlan G. Effects of obesity on respiratory resistance. Chest. 1993;103(5):1470-6.

21. Libanori HT. Estudo comparativo da resposta ao traumatismo cirúrgico em pacientes submetidos à colecistectomia por videolaparoscopia e laparotomia [dissertação]. São Paulo (SP): Universidade de São Paulo; 1996.

22. Normando VMF, Brito MVH, Araújo Jr. FA, Albuquerque BC. Repercussões do pneumoperitônio na amplitude da excursão diafragmática em suínos. J Bras Pneumol. 2006; 32(1):16-22.

23. Pereira ED, Faresin SM, Juliano Y, Fernandes AL. Fatores de risco para complicações pulmonares no pós-operatório de cirurgia abdominal alta. J Pneumol. 1996; 22(1):19-26.

24. Joris J, Kaba A, Lamy M. Postoperative spirometry after laparoscopy for lower abdominal or upper abdominal surgical procedures. Br J Anaesth. 1997;79(4):422-6.

25. Sprung J, Whalley DG, Falcone T, Wilks W, Navratil JE, Bourke DL. The effects of tidal volume and respiratory rate on oxygenation and respiratory mechanics during laparoscopy in morbidly obese patients. Anesth Analg. 2003; 97(1):278-74.

26. Helene AJ, Saad RJ,Stirbulov R. Avaliação da função respiratória em indivíduos submetidos à abdominoplastia. Rev Col Bras Cir. 2006; 33(1):45-9.

27. Chiavegato LD, Jardim JR, Faresin SM, Juliano Y. Alterações funcionais respiratórias na colecistectomia por via laparoscópica. J Pneumol .2000; 26(2):69-76.

Como citar este artigo:

Cardoso Filho GM, Diogo Filho A, Ribeiro GC. Provas de função pulmonar no pré e pós-operatório de redução gástrica por celiotomia ou videolaparoscopia. Rev Col Bras Cir. [periódico na Internet] 2008; 35(6). Disponível em URL: http://www.scielo.br/rcbc

Endereço para correspondência: Geraldo Magela Cardoso Filho

Av. João Pinheiro, 289 - Centro 38400-124 - Uberlândia - MG

E-mail: gmcardoso25@hotmail.com 